\title{
Recording 25 years of progress in Cancer Gene Therapy
}

\author{
Steven K. Libutti ${ }^{1}$
}

Received: 2 July 2019 / Accepted: 2 July 2019 / Published online: 9 July 2019

(c) Springer Nature America, Inc. 2019

At Cancer Gene Therapy (CGT), we are pleased to present this 25th Anniversary Collection, which brings together a representative sample of our best and highest-impact papers. These past 25 years have seen extraordinary progress in the fields of cancer gene therapy and cancer immunology. From the first clinical trial in the early 1990s demonstrating that genetically modified immune cells could be reintroduced into patients [1, 2], through the FDA approval of CAR-T cell therapy, the field has seen tremendous accomplishments.

From its launch by our founding editors, Drs. Robert E. Sobol and Kevin J. Scanlon, CGT has been committed to bringing its readers the most impactful original papers, reviews, and editorials. This past year, $C G T$ ranked in the top quartile in each of its subject areas and achieved its highest impact factor to date. The papers selected to make up this 25th Anniversary Collection represent the quality of work that allowed us to achieve these goals and to deliver on the vision of Drs. Sobol and Scanlan.

For example, in a paper published in 1999 entitled, Intratumoral recombinant GM-CSF-encoding virus as gene therapy in patients with cutaneous melanoma [3], Mastrangelo and colleagues reported on a clinical trial involving seven immunocompetent patients with surgically incurable cutaneous melanoma. These patients received treatment of dermal or subcutaneous metastases, with twice weekly intratumoral injections of escalating doses of a vaccinia GM-CSF recombinant virus for 6 weeks. All patients with stable or responding disease continued to receive treatment, until tumors resolved or there was evidence of progression. The authors found that this treatment was well tolerated and resulted in a dense infiltration with CD4-positive and CD8positive lymphocytes, as well as histiocytes and eosinophils in treated lesions. All seven patients developed an anti-

Steven K. Libutti

steven.libutti@cinj.rutgers.edu

1 Rutgers Cancer Institute of New Jersey, New Brunswick, NJ, USA vaccinia antibody response 14-21 days following revaccination. Despite the presence of these antibodies, the reporter gene was still expressed in patients. While the responses were mixed, this paper clearly demonstrated the feasibility of sequential administration of escalating doses of the gene carrying recombinant vaccinia virus and set the stage for similar viral vector trials that followed.

Over the years, CGT has accepted papers that have focused on vector development and methods to enhance the selectivity of either gene delivery or gene expression. In 2001, Ito and colleagues looked at heat-inducible TNF gene expression in their paper, Heat-inducible $T N F-\alpha$ gene therapy combined with hyperthermia, using magnetic nanoparticles as a novel tumor-targeted therapy [4]. Their premise was that heat-induced therapeutic gene expression would be a strategy to minimize side effects. They hypothesized that gene expression triggered by heat stress, could combine the therapeutic effects of hyperthermia and gene therapy. They combined $T N F-\alpha$ gene therapy driven by the stress-inducible promoter, gadd 153, with hyperthermia using magnetite cationic liposomes (MCLs). In a nude mouse model, MCLs induced cell death throughout the tumor following heating, using an alternating magnetic field. Heat stress resulted in a threefold increase in TNF- $\alpha$ gene expression driven by the gadd 153 promoter when compared with nonheated tumor. There was an apparent "bystander effect" as $T N F-\alpha$ gene expression was seen in areas where the hyperthermic effect was not sufficient to cause cell death. The combined treatment was successful in controlling tumor growth over a 30-day period, thus supporting the concept of this novel combination of inducible gene expression combined with nanoparticle delivery.

Another strategy for selective or targeted delivery is explored in the paper by Camp and colleagues published in 2013 and entitled, Transferrin receptor targeting nanomedicine-delivering wild-type $p 53$ gene sensitizes pancreatic cancer to gemcitabine therapy [5]. They developed liposomal nanoparticles targeted by a single-chain antibody fragment to the transferrin receptor (TfRscFv) delivering wild-type (wt) human p53 (SGT-53). They 
hypothesized that SGT-53 in combination with gemcitabine would enhance the therapeutic benefit in an in vivo metastatic pancreatic cancer model. This model was based on intrasplenic injection of murine pancreatic cancer cells, which resulted in the development of liver metastases. In order to determine nanoparticle localization, they injected $\mathrm{TfRscFv}$ with fluorescently labeled oligonucleotides (6carboxyfluorescein phosphoramidite (6FAM) ODN) by tail vein and then imaged using a Xenogen IVIS 200 scanner. SGT-53 and gemcitabine alone and in combination were administered biweekly and compared with control mice. They used survival as determined by a blinded daily assessment as their endpoint. They assessed human wt $p 53$ expression and transferrin levels in the tumors, using western blot, and quantified tumor burden by liver weight. Compared with control mice with metastatic tumors (median survival of 20 days), SGT-53, gemcitabine, and their combination demonstrated improved median survival of 29 , 30, and 37 days, respectively. The combination treatment improved median survival over a single drug treatment and decreased tumor burden. They concluded that tumor targeting liposomal-based SGT-53 nanoparticles are capable of sensitizing pancreatic cancer to conventional chemotherapy and that their unique approach had the potential to be a more effective therapy for pancreatic cancer.

As a final example of the papers selected for the 25th Anniversary Collection, Katz and colleagues explored regional delivery of CAR-T cell therapy in their paper published in 2016, Regional CAR-T cell infusions for peritoneal carcinomatosis are superior to systemic delivery [6]. Colorectal cancer (CRC) commonly spreads to the peritoneal cavity and when it does, it is accompanied by significant morbidity. While peritoneal disease can be very difficult to treat, the peritoneal cavity offers an opportunity to provide regional therapy via infusion of agents directly into the peritoneal cavity. Katz and colleagues reported on the intraperitoneal (IP) delivery of CAR-Ts for peritoneal carcinomatosis. The infusion of CAR-T into the peritoneal cavity resulted in superior protection against carcinoembryonic antigen $(\mathrm{CEA}+)$ peritoneal tumors, when compared with systemically infused CAR-Ts. CAR-T-infused IP also provided protection against IP tumor rechallenges and demonstrated an increase in effector memory phenotype over time. In addition, combinatorial IP CAR-T treatment with depleting antibodies against MDSC and Treg further improved the efficacy against peritoneal metastases. Their data support further development of combinatorial IP CAR-T immunotherapy for peritoneal malignancies and has now resulted in the initiation of a clinical trial.

It is our hope that our readers enjoy this 25th Anniversary Collection and agree that the papers presented all played an important role in adding to our understanding of how to better apply cancer gene therapy and the approaches to cancer immunotherapy. $C G T$ has been the source of information about cutting-edge science in the field of gene therapy and cancer immunology for the past 25 years. It is our intention to continue to bring you the best papers describing exciting new breakthroughs for the next 25 years and beyond.

\section{References}

1. Culver K, Cornetta K, Morgan R, Morecki S, Aebersold P, Kasid A, et al. Lymphocytes as cellular vehicles for gene therapy in mouse and man. Proc Natl Acad Sci USA. 1991;88:3155-9.

2. Morecki S, Karson E, Cornetta K, Kasid A, Aebersold P, Blaese $\mathrm{RM}$, et al. Retrovirus-mediated gene transfer into CD4+ and CD8+ human $\mathrm{T}$ cell subsets derived from tumor-infiltrating lymphocytes and peripheral blood mononuclear cells. Cancer Immunol Immunother. 1991;32:342-52.

3. Mastrangelo MJ, Maguire HC Jr., Eisenlohr LC, Laughlin CE, Monken CE, McCue PA, et al. Intratumoral recombinant GM-CSFencoding virus as gene therapy in patients with cutaneous melanoma. Cancer Gene Ther. 1999;6:409-22.

4. Ito A, Shinkai M, Honda H, Kobayashi T. Heat-inducible TNFalpha gene therapy combined with hyperthermia using magnetic nanoparticles as a novel tumor-targeted therapy. Cancer Gene Ther. 2001;8:649-54.

5. Camp ER, Wang C, Little EC, Watson PM, Pirollo KF, Rait A, et al. Transferrin receptor targeting nanomedicine delivering wildtype p53 gene sensitizes pancreatic cancer to gemcitabine therapy. Cancer Gene Ther. 2013;20:222-8.

6. Katz SC, Point GR, Cunetta M, Thorn M, Guha P, Espat NJ, et al. Regional CAR-T cell infusions for peritoneal carcinomatosis are superior to systemic delivery. Cancer Gene Ther. 2016;23:142-8. 